\begin{tabular}{l|l|c} 
Eiszeitalter u. Gegenwart & 42 & $121-133$ \\
& $8 \mathrm{Abb} ., 2 \mathrm{Tab}$.
\end{tabular}

\title{
Mittelquartäre Klimaverhältnisse und Reliefentwicklung im semiariden Becken von Vera (Südostspanien)
}

\author{
Gerd WenZens *)
}

\begin{abstract}
Upper Pleistocene, middle Pleistocene, glacis (pediments), terraces, paleoenvironment, paleoclimatology, deluvial loess, ESR, southeast Spain
\end{abstract}

Kurzfassung: Im nördlichen Teil des Beckens von Vera treten als älteste quartäre Formen villafrancazeitliche beckenrandnahe Schwemmfächer und Glacisakkumulationen auf, die an der Basis Travertine enthalten, deren ESR-Altersabschätzung bei ca. 1,6 Mill. Jahre liegt. Ein Aufleben der Beckenrandverwerfungen im Altquartär initiierte eine intensive Ausräumungsphase, so daß von dem ursprünglich das gesamte Untersuchungsgebiet einnehmenden Glacis nur noch kleinräumige Reste erhalten sind (Abb. 1). Sie überragen um bis zu $50 \mathrm{~m}$ mittelquartäre Glacisrelikte, die vor allem nördlich von Cuevas del Almanzora das Landschaftsbild bestimmen. Am Einschnitt des Flusses bestehen sie aus ca. $35 \mathrm{~m}$ mächtigen Ablagerungen, in denen vereinzelte Grobblöcke auftreten. Diese zwischen 3 und 4,5 m Längsachse erreichenden kantigen Bruchstücke entsprechen in ihrem Habitus Driftblöcken und legen einen Transport auf Eisschollen nahe. Hinweise auf eine intensive winterliche Abkühlung in diesem küstennahen Raum geben Hangschuttdecken, deren Einregelungsmessungen einen gelisolifluidalen Transport anzeigen. Da sie vereinzelt an ihrer Basis mit den liegenden sandig-schluffigen Ablagerungen verwürgt sind, ist von einer bedeutenden Frosteindringtiefe auszugehen. Diese Schlußfolgerung wird durch den Fund eines ca. $50 \mathrm{~cm}$ Durchmesser erreichenden Blocks aus Sand gestützt, dessen Rundung nur durch einen längeren fluviatilen Transport im gefrorenen Zustand erklärbar ist. Die aufgeführten Merkmale sprechen insgesamt für eine winterliche Temperaturabsenkung von $12-15^{\circ} \mathrm{C}$. In die Glacisablagerungen sind z. T. mehrere Meter mächtige äolische Sedimente eingeschaltet, die zwar lokal hohe sandige Beimengungen aufweisen, in der Regel aber zwischen 50 und $75 \%$ Schluff enthalten. Sie werden als Deluviallösse und Indikatoren kalt-arider Klimabedingungen interpretiert.

Mit diesem frühmittelquartären Niveau ist die Entstehung weiträumiger Glacisflächen beendet; im folgenden schneiden sich die Flüsse ein und bewirken die partielle Aus-

*) Anschrift des Verfassers: Prof. Dr. G. Wenzens, Geographisches Institut der Heinrich-Heine-Universität Düsseldorf, Universitätsstr. 1, D - 4000 Düsseldorf 1. räumung der Akkumulationen. Nur längs des Río Almanzora lassen sich noch Terrassenniveaus aus dem Mittel- und Jungquartär ausgliedern.

[Mid-quaternary climatic conditions and relief development in the Vera basin (southeast Spain)]

Abstract: In the northern part of the Vera basin the oldest quaternary forms are alluvial fans and glacis plains near the base of the mountains. Travertine layers at their bottom are dated by ESR as approximately 1.6 mio years old.

A revival of tectonic activities in the transition zone between basin and mountains during the early quaternary caused a period of intense erosion so that today there appear only small relics of the glacis which once covered all the study area (fig. 1). They are situated up to 50 metres above relics of mid-pleistocene glacis dominating the landscape especially north of Cuevas del Almanzora. At the river's cut they consist of about 35 metres of accumulations which include few boulders. As to their appearance these blocks, angular and reaching 3 to 4.5 meters of longitudinal axis are similar to drift blocks and seem to have been transported on sheets of ice.

Talus deposits, whose orientation measurements indicate a movement by gelifluction, also point to intense falls of the winter temperature in this coastal region. As their base shows some slight cryoturbations with the lying silty-sandy sediments the depth of frost penetration must have been considerable. This conclusion is supported by a boulder of solidified sand, $0.5 \mathrm{~m}$ in diameter; its roundness can only be explained by its frozen state during a long-lasting fluvial transport.

So there is every reason to believe that winter temperatures have been $12^{\circ}$ to $15^{\circ} \mathrm{C}$ lower than today. The glacis sediments partially include aeolian sediments reaching several metres of thickness and though locally showing considerable sandy components they generally contain $50-75 \%$ of silt. They are interpreted as deluvial loesses and indications of a cold-arid climate. 
The early-mid-quaternary glacis level marks the end of the formation of widespread glacis plains. In the following morphogenetic cycle the rivers cut and cause the partial erosion of the sediments. It is only along the Almanzora river that mid- and late-quaternary terrace levels can be found.

\section{[Conditions climatiques et dévelopment de relief quaternaire moyen dans le bassin de Vera (Espagne du sud-est)]}

Résumé: Dans la partie septentrionale du bassin de Vera les plus anciennes formations du quaternaire sont des cônes alluviaux villefranchiens se trouvant près du bord du bassin et des accumulations de glacis qui, à la base, contiennent du travertin datant vers 1,6 million d'ans (par ESR). Une revivification des failles près du bord du bassin initiait une phase d'enlèvement intensive au quaternaire ancien de manière qu'il ne persiste que des restes peu considérables du glacis ayant couvert avant toute la région de l'étude (fig. 1). Ils dépassent les vestiges quaternaires moyens de 50 mètres environ qui déterminent le cycle de l'ensemble du relief avant tout au nord de Cuevas del Almanzora. A l'incision de la rivière ils se composent de dépots d'une épaisseur de 35 mètres contenant des gros blocs isolés. Ces fragments anguleux atteignant une axe longitudinal entre 3 et 4,5 mètres ressemblent à des "Driftblöcke" et permettent de supposer un transport par des blocs de glaces. Des éboulis dont des mesures de réglage indiquent un transport gélisolifluvial signalent un refroidissement hivernal intensif dans cette région près de la côte. Comme ils sont sporadiquement cryoturbés avec des sédiments sableux et silteux, il faut supposer une profondeur considérable de pénétration du gel. Cette conclusion est confirmée par un bloc atteignant vers $50 \mathrm{~cm}$ de diamètre qui consiste en sable légèrement consolidé dont la rondeur s'explique seulement par un long transport fluvial en état gelé. Les indices cités soulignent la supposition d'une baisse des températures hivernales de 12 à 15 degrés $C$. Dans les sédiments du glacis apparaissent partiellement plusieurs mètres d'accumulations éoléennes qui de temps en temps contiennent des parties sableuses, mais en général se composent de 50 à $75 \%$ de silt. Elles sont classifiées comme loess déluvial et interprétées comme indicateurs de conditions climatiques froides-arides. La formation de vastes plaines de glacis se termine par ce niveau du quaternaire moyen inférieur. Dans la suite les rivierès s'entaillent et provoquent l'érosion partielle des sédiments. Seul le long du Río Almanzora il est toujours possible de différencier des niveaux de terrasse du quaternaire moyen et inférieur.

\section{Problemstellung}

Die Erforschung der quartären Reliefentwicklung in Südostspanien beinhaltet in erster Linie die Frage, in welcher Weise sich die Glaziale und Interglaziale der gemäßigten Breiten in dieser fast ariden Region ausgewirkt haben. Spätestens zu Beginn der 70er Jahre mußten die Vorstellungen der "Pluvialhypothese" revidiert werden. Sowohl das Torfprofil von Padul als auch die Molluskenfauna in Lössen ergab für das
Maximum des Würm-Glazials ein kalt-arides Klima. Allerdings bestehen bezüglich des Ausmaßes der Temperaturerniedrigung während der letzten Kaltzeit unterschiedliche Auffassungen.

Im Vergleich zu den Kenntnissen über die Klimaentwicklung des Jungpleistozäns liegen bezüglich der mittelquartären Klimaverhältnisse in Südspanien nur vage Vorstellungen vor (LHËNAFF 1986). Insbesondere ist umstritten, ob in den Glazialen eher periglaziale oder semiaride Bedingungen die Morphodynamik beeinflußt haben (COUVREUR \& RAYNAL 1981: 49). Während Kryoturbationen in den mittelquartären Ablagerungen zentralspanischer Flußterrassen (BROSCHE 1978a: 192f.) auf eine insgesamt stärkere Abkühlung zur Zeit der älteren Glaziale schließen lassen, geht DUMAS (1979: 36) in Südostspanien nur von einer mäßigen Abkühlung in den älteren Kaltzeiten aus und sieht vor allem in der Verringerung der Niederschläge den Auslöser der Glacisbildung. Nach BOUSQUET (1986: 32) dagegen wird die mittelquartäre Reliefentwicklung in Spanien in erster Linie von der tektonischen Aktivität geprägt. Ein Hauptanliegen dieser Studie ist es, Geländebefunde, wie Sandgerölle, Driftblöcke, Hangschuttdecken mit Merkmalen von Verwürgungen sowie Deluviallösse in mittelquartären Glacisablagerungen auf ihre klimatische Aussagekraft hin zu analysieren. Im Zusammenhang mit der Rekonstruktion der mittelquartären Glacis- und Flußterrassenbildung soll auch die Bedeutung der Tektonik für die Reliefentwicklung untersucht werden.

\section{Physisch-geographische Ausstattung des Untersuchungsgebietes}

Das Untersuchungsgebiet (Abb. 1) befindet sich im Ostteil der Betischen Kordillere. Im Zusammenhang mit der jungtertiären Heraushebung dieses Gebirgssystems entstanden tektonische Senken, die im Neogen mit marinen Sedimenten, insbesondere Mergeln, verfüllt wurden (VÖLK 1967, HARVEY 1987). Innerhalb dieser Senkungsräume bildet das Untersuchungsgebiet den nördlichen Teil des halbkreisförmigen, unmittelbar an das Mittelmeer grenzenden Beckens von Vera. Seine Gebirgsumrahmungen bestehen aus Deckenkomplexen unterschiedlichen Metamorphosegrades. Das Becken von Vera wird von drei Flußsystemen durchquert, dem Río de Aguas im Süden, dem Río Antas im Zentrum und dem Río Almanzora im Norden. Dieser zählt mit über $100 \mathrm{~km}$ Länge und einem über $2600 \mathrm{~km}^{2}$ großen Einzugsgebiet zu den bedeutendsten Flüssen in Südostspanien. Er entspringt in der über $2000 \mathrm{~m}$ hohen Sierra de los Filabres und fließt an ihrem Nordfuß in einer West-Ost streichenden Senke. Eine tektonische Schwächezone in 


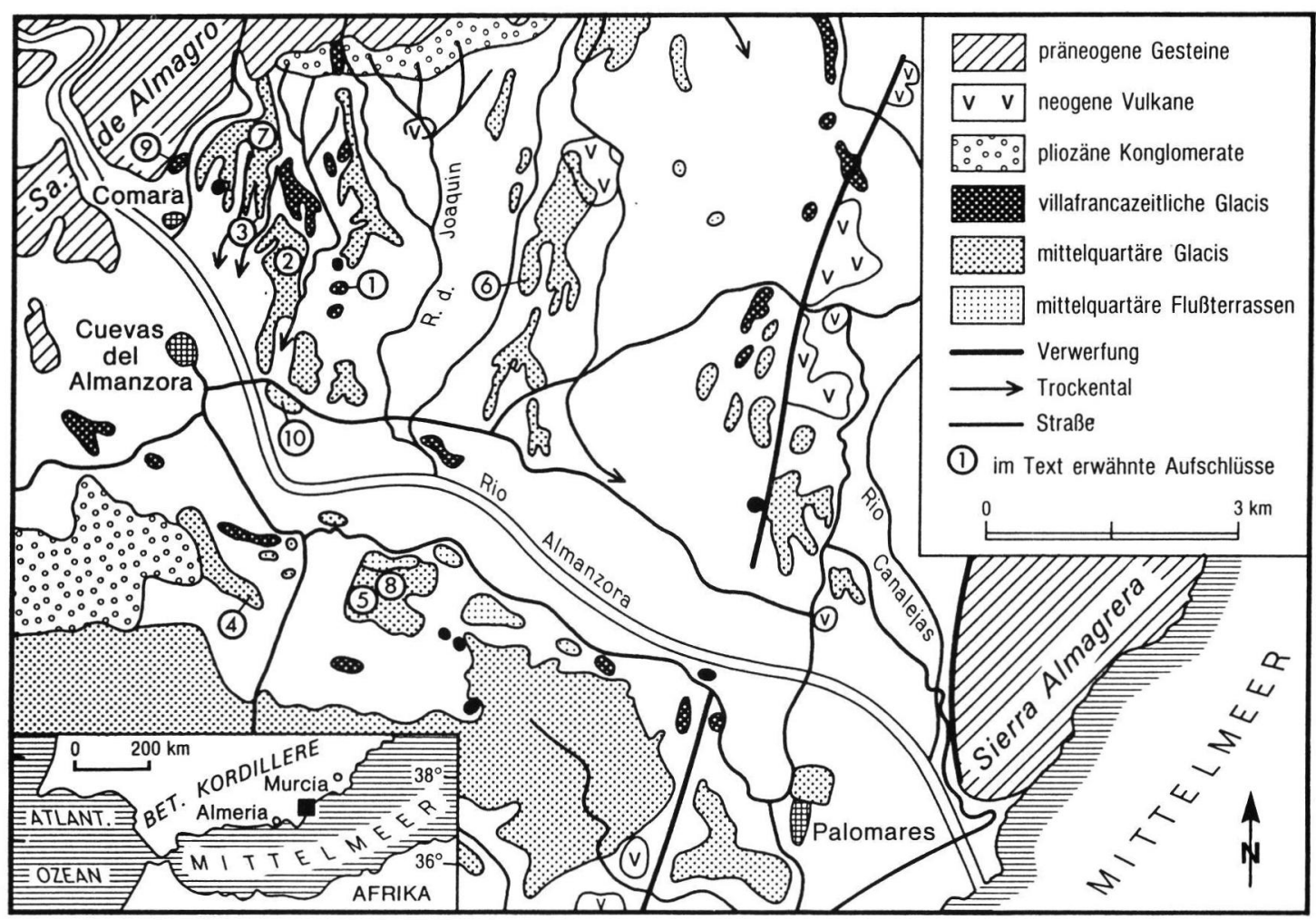

Abb. 1: Mittelquartäre und villafrancazeitliche Glacis und Flußterrassen sowie Lage der Aufschlüsse im Untersuchungsgebiet.

der $700 \mathrm{~m}$ ü. NN erreichenden und vorwiegend aus Kalken und Dolomiten aufgebauten Sierra de Almagro nutzend, durchbricht er diese in einem steilen Engtal und tritt in $80 \mathrm{~m}$ ü. NN in das Tertiärbecken von Vera ein. Vom 250-300 m hohen Gebirgsfuß bis zum ca. $12 \mathrm{~km}$ entfernten Mittelmeer läßt sich ein System von Schwemmfächern, Glacisflächen und Flußterrassen differenzieren. Sie sind durch teilweise bis zum Beckenrand sich ausdehnende Austäumungsbuchten in meist schmale Riedel aufgelöst, woraus ersichtlich wird, daß sowohl Erosions- als auch Akkumulationsphasen die quartäre Reliefentwicklung dieses mit $200-250 \mathrm{~mm}$ Jahresniederschlag fast ariden Beckens bestimmen.

Für den am Eintritt des Río Almanzora in das Becken liegenden Ort Cuevas del Almanzora ( $90 \mathrm{~m}$ ü. NN) gibt VÖLK (1973: 269) $254 \mathrm{~mm}$, FerRE BuENO (1979, 48) $218 \mathrm{~mm}$ Jahresniederschlag an.

Wie in allen Trockengebieten treten auch hier große jährliche Schwankungen auf: Mit $498 \mathrm{~mm}$ wurden 1950 maximale, mit nur $54 \mathrm{~mm}$ im Jahre $1961 \mathrm{mini}-$ male Jahresniederschläge gemessen (VÖLK 1973, 270). Die Niederschläge mit geringer Intensität in Form von Sprühregen bleiben morphologisch wirkungslos; für die fluviale Dynamik sind lediglich die Starkregen von
Bedeutung; hinzu kommen Überschwemmungen großen Ausmaßes. So haben seit 1870 elf bedeutende Überflutungen (HOFFMANN 1988: 9f.) stattgefunden. Nach THORNes (1976) fielen im Einzugsgebiet des Río Almanzora am 18. und 19. Oktober 1973 innerhalb von 36 Stunden bis zu $250 \mathrm{~mm}$ Niederschlag, und es kam am Unterlauf des Flusses zu einer Schlammflut, die erhebliche Schäden anrichtete.

In mehreren Kiesgruben sind die holozänen Flußablagerungen aufgeschlossen. In ihrer Zusammensetzung spiegeln sich die in den letzten 120 Jahren als katastrophale Hochwässer des Río Almanzora dokumentierten, bis zu $15 \mathrm{~m}$ hohen Fluten nicht in Form markanter Grobgeröllhorizonte bzw. -nester wider. Vielmehr haben die Fluten jeweils nach Phasen weitgehend fehlender Akkumulation, während derer der Almanzora als zwar ständig fließendes, aber sehr wenig Wasser führendes schmales Gerinne ausgebildet war, höchstens Sedimente der Geröllfraktion transportiert. Die Ablagerungssequenz ist also durch eine Vielzahl von Hochwässern aufgebaut worden. Die holozänen Sedimente zeigen die Schwierigkeiten auf, allein aus den Flußablagerungen weitreichende Schlußfolgerungen auf die jeweiligen Klimagegebenheiten ziehen zu wollen. 
Tab. 1: Ausgewählte Temperaturwerte der Stationen Almería ( $7 \mathrm{~m}$ ü. M.; $36^{\circ} 50^{\prime} \mathrm{N} / 2^{\circ} 28^{\prime} \mathrm{W}$ ) und Murcia $44 \mathrm{~m}$ ü. M.; $37^{\circ} 59^{\prime} \mathrm{N} / 1^{\circ} 08^{\prime} \mathrm{W}$ )

\begin{tabular}{|c|c|c|c|c|c|c|c|c|c|c|c|c|c|}
\hline & \multicolumn{13}{|c|}{ Mittlere Temperaturen in ${ }^{\circ} \mathrm{C}$} \\
\hline & $\mathrm{J}$ & $\mathrm{F}$ & M & A & M & $\mathrm{J}$ & $\mathrm{J}$ & A & $\mathrm{S}$ & $\mathrm{O}$ & $\mathrm{N}$ & $\mathrm{D}$ & Jahr \\
\hline Almería & 11,7 & 11,8 & 14,1 & 16,1 & 18,4 & 22,0 & 24,7 & 25,3 & 23,4 & 19,4 & 15,6 & 12,8 & 18,0 \\
\hline \multirow[t]{3}{*}{ Murcia } & 10,0 & 11,2 & 14,0 & 16,2 & 19,4 & 23,8 & 26,4 & 26,4 & 23,7 & 18,9 & 14,3 & 11,4 & 18,0 \\
\hline & \multicolumn{13}{|c|}{ Mittleres Minimum der Temperaturen in ${ }^{\circ} \mathrm{C}$} \\
\hline & $\mathrm{J}$ & $\mathrm{F}$ & M & A & M & $\mathrm{J}$ & $\mathrm{J}$ & A & S & $\mathrm{O}$ & $\mathrm{N}$ & $\mathrm{D}$ & Jahr \\
\hline Almería & 4,2 & 4,5 & 6,5 & 8,9 & 10,9 & 14,8 & 17,4 & 18,1 & 15,8 & 11,4 & 8,2 & 5,2 & 10,5 \\
\hline \multirow[t]{3}{*}{ Murcia } & $-1,1$ & $-0,7$ & 2,5 & 5,7 & 8,2 & 13,4 & 16,0 & 15,7 & 12,9 & 6,9 & 3,2 & 0,6 & 6,9 \\
\hline & \multicolumn{13}{|c|}{ Absolutes Minimum der Temperaturen in ${ }^{\circ} \mathrm{C}$} \\
\hline & $\mathrm{J}$ & $\mathrm{F}$ & M & A & M & $\mathrm{J}$ & $\mathrm{J}$ & A & $\mathrm{S}$ & $\mathrm{O}$ & $\mathrm{N}$ & $\mathrm{D}$ & \\
\hline Almería & 1,9 & 0,2 & 2,6 & 5,3 & 8,2 & 12,7 & 14,6 & 15,5 & 10,1 & 7,6 & 4,5 & 2,5 & \\
\hline Murcia & $-5,0$ & $-10,0$ & $-3,0$ & 2,0 & 4,0 & 11,0 & 9,8 & 11,6 & 8,0 & $-4,2$ & $-1,4$ & $-5,0$ & \\
\hline
\end{tabular}

Quelle: MüLler, M. (1979: 83 u. 84)

Für die Interpretation der kaltzeitlichen Klimabedingungen, die aus den korrelaten Ablagerungen rekonstruiert werden sollen, sind vor allem die Temperaturwerte von Bedeutung.

$\mathrm{Da}$ eine nur 6 Jahre umfassende Temperaturmessung für Cuevas del Almanzora vorliegt, sollen die entsprechenden Werte der ca. $100 \mathrm{~km}$ entfernten Klimastationen Murcia im Norden und Almería im Süden (s. Abb. 1) berücksichtigt werden.

Obwohl die mittleren Monatstemperaturen beider Stationen weitgehend übereinstimmen, ist das mittlere Minimum der Wintermonate in Murcia um ca. $5^{\circ}$ niedriger und erreicht hier im Januar und Februar weniger als $0^{\circ} \mathrm{C}$. Noch extremer sind die Unterschiede der absoluten Minima im Winterhalbjahr.

Im folgenden soll versucht werden, die morphologische Wirkung von mittelquartären Klimaschwankungen mit Hilfe der am Unterlauf des Río Almanzora vorliegenden Akkumulationsformen zu erfassen und zu versuchen, aus dem jeweiligen Sedimentationsmilieu Rückschlüsse auf die zugehörigen Klimabedingungen zu ziehen, wobei auch die in den Sedimenten enthaltenen äolischen Akkumulationen zur Rekonstruktion der Klimaverhältnisse herangezogen werden. Bodenbildungen sowie die in unterschiedlicher Ausbildung entwickelten Kalkkrusten bleiben dagegen hier unberücksichtigt.

\section{Stand der Forschung}

VÖLK (1979) hat im Becken von Vera 4 Glacisterrassen aus dem Mittelquartär beschrieben. Jeder einzelne Glaciskörper ist durch eine fluviatile Talweg-Verschüttung und anschließende fanglomeratische GlacisAkkumulation aufgebaut. Da die Sedimentation bei einer episodisch-torrentiellen Pluvialität im frühen Interglazial erfolgt und das semiaride Haupt- und Spätglazial durch eine morphodynamische Stabilität gekennzeichnet ist, ist der Einfluß kaltzeitlicher Klimabedingungen auf die Morphodynamik unbedeutend. Nach VÖLK (1979: 87) sind äolische Prozesse und Frostschuttbildung in dieser Zeit höchstens im Gebirge möglich.

FERRE BUENO (1979: 108f.) hat im Untersuchungsgebiet insgesamt 5 Niveaus ausgegliedert, wobei er zwischen 3 Glacisflächen, einer Flußterrasse und dem rezenten Flußniveau unterscheidet. Das höchstgelegene Glacis stellt er in das Villafranca, das folgende, ca. 10-20 m tiefer gelegene, das in Flußnähe in eine Terrasse übergeht, in die Rißkaltzeit. Aus dem dazwischenliegenden Zeitraum werden keine morphologischen Formen beschrieben. Die Problematik des Einflusses quartärer Klimaschwankungen auf die Morphodynamik bleibt in dieser Art unberücksichtigt.

In mehreren Arbeiten hat HARVEY $(1978,1987)$ die Reliefentwicklung in Südostspanien untersucht und 
vor allem den Einfluß der Tektonik auf die unterschiedliche Morphogenese dieses Raumes betont. Die Hauptablagerungsperioden stellt HARVEY (1987: 206) in "kalt-trockene Glaziale”, ohne allerdings die Transport- und Sedimentationsbedingungen der teilweise über $20 \mathrm{~m}$ mächtigen Akkumulationen zu benennen.

Erste Versuche, in Südspanien Hinweise auf das Ausmaß der Temperaturabsenkung während des Würms zu ermitteln, haben BRUNNACKER \& LOŽEK bereits 1969 vorgenommen. Die Untergrenze der Periglazialstufe setzen sie bei $700 \mathrm{~m}$ ü. NN an und schließen daraus auf eine Temperaturabsenkung um $10^{\circ} \mathrm{C}$, später hielt BRUNNACKER (1979: 112) auch eine Temperaturdepression von $10-12^{\circ} \mathrm{C}$ im Vergleich zu heute für möglich. BROSCHE (1978c: 236) nimmt für Südspanien ebenfalls eine Temperaturdepression in den Winter- und Frühjahrsmonaten von ca. $10^{\circ} \mathrm{C}$ an und schließt aufgrund von Moränenfunden in der Sierra de la Sagra sogar eine größere Temperaturerniedrigung nicht aus (BROSCHE 1978a: 196).

BRUNNACKER \& LOŽEK $(1969,298)$ erwähnen Deluviallößvorkommen bei dem ca. $12 \mathrm{~km}$ nordwestlich von Cuevas del Almanzora gelegenen Ort Huércal Overa ( $280 \mathrm{~m}$ ü. NN). Sie setzen die Untergrenze des Lösses in diesem Raum bei $50-100 \mathrm{~m}$ ü. NN an (BRUNNACKER \& LOŽEK 1969: 308). BLÜMEL (1981: 138) fiel bei der Untersuchung einer Kalkkruste $2,5 \mathrm{~km}$ nördlich von Garrucha in $20 \mathrm{~m}$ ü. NN auf, daß der „Habitus des feinkörnigen Lösungsrückstandes . . . dem des Lösses vom Profil Ebro (Kap. 6.1.1.2.) und Librilla" ähnelt.

Dumas (1979: 232f.), der lediglich von kurzfristigen Temperaturabsenkungen um $6-8^{\circ} \mathrm{C}$ während des Würms ausgeht, bezweifelt den periglazialen Lößcharakter der von BRUNNACKER \& LOŽEK beschriebenen Deluviallösse. Er schließt zwar grundsätzlich die Existenz von Lössen nicht aus, hält diese jedoch für Lösse des ariden Typs.

\section{Die villafrancazeitliche Reliefentwicklung im Raum Cuevas del Almanzora}

Mit der an der Wende Pliozän/Pleistozän beginnenden Hebung des Beckenrandes zog sich das Meer allmählich zurück. Gleichzeitig setzte am Beckensaum die Akkumulation von Schwemmfächern ein. Diese vor allem nordwestlich Cuevas del Almanzora erhaltenen Ablagerungen weisen an der Basis bis zu $1 \mathrm{~m}$

1 Ich danke Herrn Univ. Doz. Dr. U. RADTKE, Düsseldorf, für zahlreiche ESR-Datierungen. mächtige limnische Kalke auf, deren ESR ${ }^{1}$-Altersabschätzung bei über 2,358 Mill. Jahre liegt. Die bis zu $50 \mathrm{~m}$ mächtigen Schwemmfächer werden als Äquivalente der zentralspanischen Rañaformation gedeutet (WENZENS \& ROMMERSKIRCHEN 1979: 101f.) und in das untere bis mittlere Villafranca gestellt (WENZENS 1992, 178). Im Anschluß an ihre Sedimentation fand eine weitere Heraushebung statt, in deren Verlauf sich das Meer aus dem gesamten Beckenbereich zurückzog. Während die villafrancazeitlichen Schwemmfächer von nur kurzen Gerinnen sedimentiert wurden, die ein sehr kleines Einzugsgebiet hatten, entwickelten sich nun die heutigen Barrancas, die teilweise weit in die Sierra de Almagro zurückgreifen. Mit der nachlassenden Transportkraft am Gebirgsfuß kam es innerhalb des Beckens zu mächtigen Akkumulationen, die sich miteinander verzahnen und im Gegensatz zu den Schwemmfächern aufgrund ihrer flächenhaften Verbreitung und ihres geringen Gefälles $\left(<3^{\circ}\right)$ als Glacis bezeichnet werden.

Im Raum Cuevas del Almanzora sind nur noch isolierte Relikte dieses Niveaus, so z. B. die $2 \mathrm{~km}$ nordöstlich des Ortes liegenden „Tres Cabezos” (Abb. 1, Aufschluß 1) erhalten. Größere Vorkommen treten im Wasserscheidenbereich zur Rambla de las Gachas in der Nordostecke des Beckens auf, wo für Travertine, die in die Basis eingelagert sind, ein durchschnittliches ESR-Alter von 1,635 Mill. Jahre ermitteltwurde (WENZENS 1992, 180). Dieses in das mittlere bis obere Villafranca eingestufte Niveau weist als charakteristisches Merkmal an der Basis stets ein mehrere Meter mächtiges Konglomerat auf. Am Beispiel der Tres Cabezos soll die Zusammensetzung dieses Glacisniveaus vorgestellt werden. Die über $20 \mathrm{~m}$ mächtigen Sedimente setzen mit einem $8 \mathrm{~m}$ mächtigen, in einer Kiesmatrix zu einem Konglomerat verfestigten Geröllpaket ein. Die folgenden $2-3 \mathrm{~m}$ mächtigen $\mathrm{Ge}$ rölle sind weniger stark inkrustiert und enthalten auffallend viele gerundete bis kantengerundete Blöcke von über $1 \mathrm{~m}$ Durchmesser. Der größte Block erreicht eine Längserstreckung von $3 \mathrm{~m}$. Dagegen bestehen die hangenden Ablagerungen aus dicht gepackten gerundeten Geröllen, wobei die Komponenten zwischen 8 und $12 \mathrm{~cm}$ Durchmesser überwiegen. Nur einzelne Sandlinsen bzw. maximal $1 \mathrm{~m}$ mächtige Sandschichten sind zwischengeschaltet; den Abschluß bilden mit fester Kalkkruste ummantelte Gerölle. Die Rundung der im Extremfall bis zu $3 \mathrm{~m}$ im Durchmesser erreichenden Blöcke weist auf ihren fluviatilen Transport hin. Die Bewegung dieser Großblöcke setzt eine zumindest zeitweise kräftige Abflußdynamik voraus, die synsedimentäre Kalkverfestigung fand durch Kalkausscheidung infolge von Verdunstung während abflußschwacher Perioden statt. Da die holozänen Ablagerungen des Río Almanzora weder inkrustiert sind noch Blöcke mit mehr als $1 \mathrm{~m}$ Durchmesser enthalten, 
reicht ein Analogieschluß von den Abflußbedingungen während der rezenten katastrophalen Hochwässer auf die villafrancazeitlichen Verhältnisse nicht aus, um die Zusammensetzung dieser Sedimente zu erklären.

\section{Die mittelquartäre Reliefentwicklung im Raum Cuevas del Almanzora}

Das villafrancazeitliche Niveau überragt Relikte einer weiteren Glacisfläche (Abb. 2) um bis zu $60 \mathrm{~m}$. Dieses Niveau bestimmt knapp $2 \mathrm{~km}$ nördlich von Cuevas del Almanzora weiträumig die Oberfläche. Es setzt an der nördlichen Gebirgsumrahmung in ca. $180 \mathrm{~m}$ ü. NN ein, fällt mit $2-3^{\circ}$ Neigung zum Beckenzentrum bis auf ca. $135 \mathrm{~m}$ ü. NN ab und geht in Flußnähe lokal mit einer deutlichen Stufe in ein ca. $120 \mathrm{~m}$ ü.M. hohes Niveau über. Das Glacis wird hier von einem knapp $20 \mathrm{~m}$ mächtigen Feinsedimentpaket gebildet, das einem Geröllkörper in ca. $100 \mathrm{~m}$ ü. NN aufliegt. Am Steilabfall zur holozänen Talaue, wo die Feinsedimente fehlen, tritt der Geröllkörper als selbständiges Niveau in Erscheinung. Die die Steilstufe zum Río Almanzora aufbauenden, bis zu $25 \mathrm{~m}$ mächtigen Ablagerungen bestehen aus fluvial transportierten Geröllen, Kiesen und Sanden. In die vorwiegend weni- ger als $30 \mathrm{~cm}$ im Durchmesser erreichenden Gerölle sind einzelne, kantige Grobblöcke eingeschaltet. Im Extremfall wurde eine 4,5 m lange, knapp $1 \mathrm{~m}$ hohe Gesteinsplatte gefunden (Abb. 1, Aufschluß 2; Abb. 3). Sie befindet sich unmittelbar westlich des $178 \mathrm{~m}$ hohen "Cabezo" und somit in gleicher Entfernung zum Beckenrand wie der $3 \mathrm{~m}$ große gerundete Block des villafrancazeitlichen Glacis. Während die Blöcke in diesem Niveau stets Rundungsmerkmale aufweisen und häufig lagenweise auftreten, sind in mittelquartären Glacisrelikten angetroffene isolierte Großblöcke in der Regel noch nicht einmal kantengerundet und oft von plattiger Gestalt. Es ist daher naheliegend, davon auszugehen, daß es sich bei den über $2 \mathrm{~m}$ im Durchmesser erreichenden kantigen Komponenten um Driftblöcke handelt, die auf Eisschollen transportiert wurden.

In diesem Fall hätte eine winterliche Temperaturabsenkung von ca. $12^{\circ} \mathrm{C}$ in dem über $2000 \mathrm{~m}$ ü. NN erreichenden Einzugsgebiet die entsprechenden Voraussetzungen hervorgerufen. Mit der Frühjahrsschneeschmelze ist infolge der Verringerung der Evapotranspiration von einer erheblichen Erhöhung der Abflußmengen auszugehen, so daß Eisschollen in relativ kurzer Zeit bis in das Becken von Vera transportiert werden konnten.

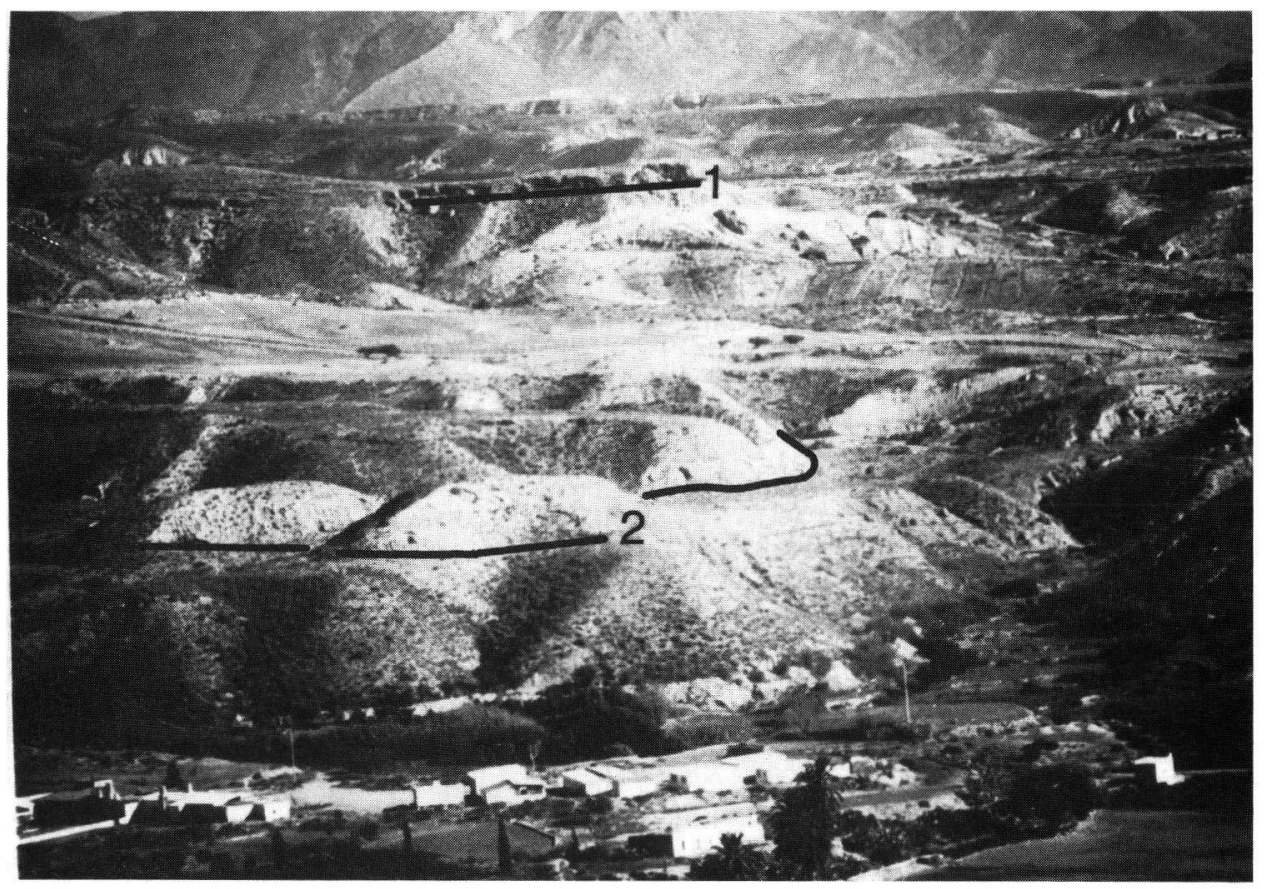

Abb. 2: Mittelquartäre und villafrancazeitliche Glacis

1 = Unterkante des villafrancazeitlichen Akkumulationskörpers 2 = Auflagerung der mittelquartären Deluviallösse 


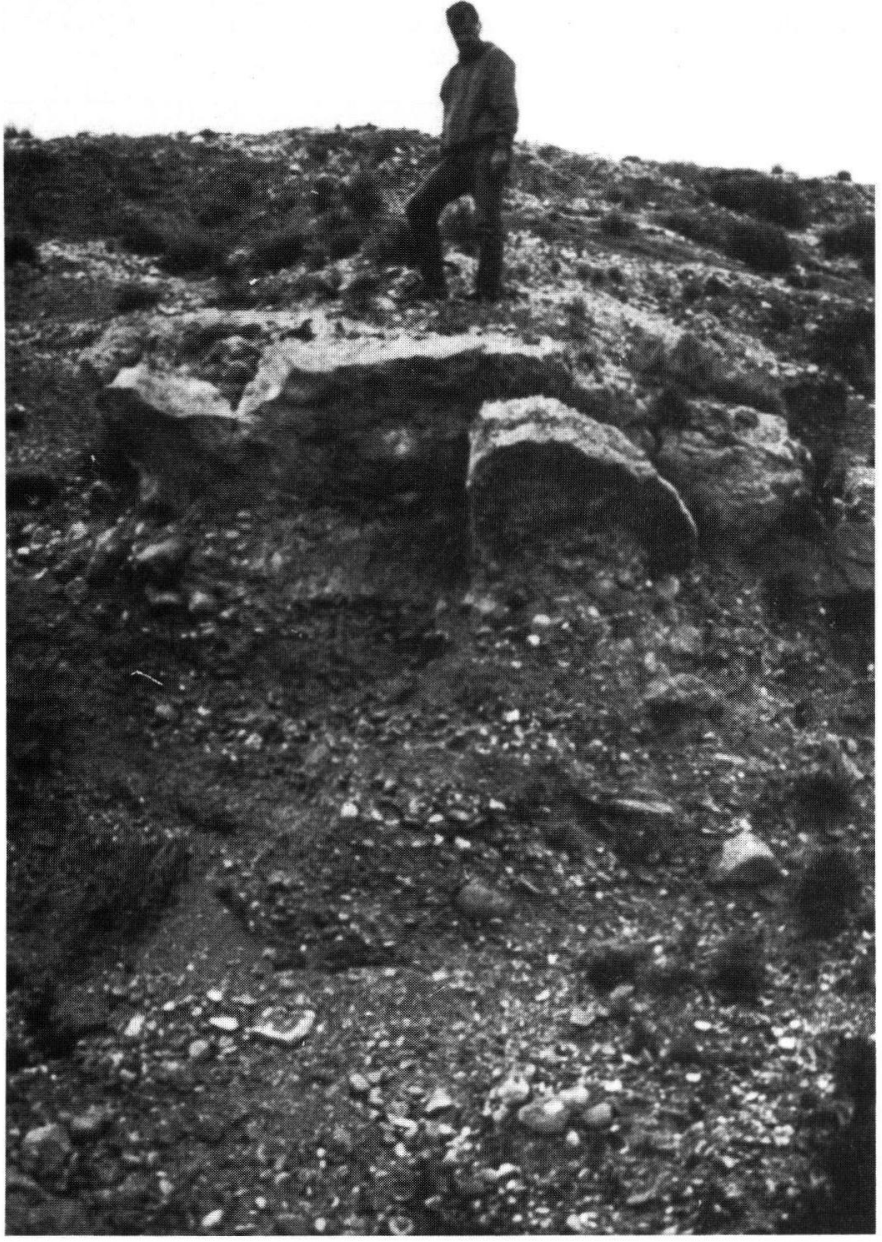

Abb. 3: Driftblock im mittelquartären Akkumulationskörper - Aufschluß 2
Nordöstlich von Comara (vgl. Abb. 1) setzt mit einer scharfen Grenze über den oben beschriebenen Ablagerungen ein $16-20 \mathrm{~m}$ mächtiges Feinsedimentpaket (Abb. 1, Aufschluß 3; Abb. 4) ein. Zum überwiegenden Teil besteht es aus sandigen Schluffen, in die einzelne Sand- und Calichelagen zwischengeschaltet sind. VÖLK (1979: 19) interpretiert sie als "hier vorwiegend schluffige Schwemmfächer-Sedimente”. Da sie jedoch mit scharfer Grenze über den liegenden Geröllen einsetzen, die schluffigen Partien keine Schichtung aufweisen und völlig steinfrei sind, ist zu überprüfen, inwieweit sie äolische Ablagerungen darstellen. Die Korngrößenanalysen (Tab. 2) stützen diese Deutung.

Mit 40-50\% und in einzelnen Lagen sogar über $60 \%$ weisen sie ein deutliches Maximum in der Mittel- und Grobschlufffraktion auf. Da jedoch auch der Sandanteil mit $25-42 \%$ recht hoch ist, werden sie als stark sandiger Deluviallöß angesprochen, der in Wechsellagerung mit Bodenbildungen auftritt. Als
Liefergebiet der äolischen Sedimente kommt das jahreszeitlich ausgetrocknete, unmittelbar südlich angrenzende breite Talbett des Almanzora infrage, was auch den relativ hohen Sandgehalt erklärt. Die inselartige Verbreitung vor dem $187 \mathrm{~m}$ hohen villafrancazeitlichen Rücken läßt sich gut mit den dominierenden Winden aus SSW und SW (HoFFMANN 1988: 10) vereinbaren. Wesentlich geringmächtiger, aber in der Korngrößenzusammensetzung mitteleuropäischen Lössen vergleichbar, sind die in den weiteren mittelquartären Glacisresten (Abb. 1, Aufschluß 4-6; Tab. 2, Abb. 5) enthaltenen Deluviallösse. Im Aufschluß 4 liegt eine $4 \mathrm{~m}$ mächtige Schluffablagerung vor, deren äolische Entstehung zwingend ist, weil das eng begrenzte Einzugsgebiet ausschließlich aus pliozänen bzw. villafrancazeitlichen Konglomeraten ohne jegliche Feinsedimenteinschaltung besteht.

Die Mittel- und Grobschlufffraktion liegt zwischen 53 und $60 \%$, der Feinsandanteil schwankt zwischen 5 und $25 \%$; der Mittelsandanteil ist gering und der 
Tab. 2: Korngrößenzusammensetzung (in \%), Kalkgehalt und Bodenfarbe nach Munsell Soil Color Charts (1954) ausgewählter äolischer Sedimente (Lage der Aufschlüsse s. Abb. 1)

\begin{tabular}{|c|c|c|c|c|c|c|c|c|c|}
\hline \multirow[t]{2}{*}{$\begin{array}{l}\text { Aufschluß } \\
\text { (s. Abb. 1) }\end{array}$} & \multirow{2}{*}{$\begin{array}{c}\text { Ton } \\
11\end{array}$} & \multicolumn{3}{|c|}{$\begin{array}{l}\text { Fein-Mittel-Grob- } \\
\text { Schluff }\end{array}$} & \multicolumn{3}{|c|}{$\begin{array}{l}\text { Fein-Mittel-Grob- } \\
\text { Sand }\end{array}$} & \multirow{2}{*}{$\begin{array}{c}\mathrm{CaCO}_{3} \\
\text { in } \% \\
10\end{array}$} & \multirow{2}{*}{$\begin{array}{l}\begin{array}{c}\text { Bodenfarbe } \\
\text { trocken }\end{array} \\
7,5 \text { YR } 8 / 2\end{array}$} \\
\hline & & 2 & - & 62 & 22 & 2 & 1 & & \\
\hline \multirow[t]{3}{*}{3} & 9 & - & 27 & 26 & 13 & 15 & 10 & 17 & 7,5 YR $8 / 2$ \\
\hline & 13 & 8 & 18 & 26 & 21 & 9 & 5 & 13 & 7,5 YR $8 / 2$ \\
\hline & 11 & 8 & 16 & 38 & 26 & 1 & - & 22 & $2,5 \mathrm{Y} 7 / 2$ \\
\hline \multirow[t]{3}{*}{4} & 21 & 14 & 31 & 25 & 8 & - & - & 20 & $2,5 Y 7 / 4$ \\
\hline & 19 & 15 & 34 & 26 & 6 & - & - & 23 & $2,5 \mathrm{Y} 7 / 2$ \\
\hline & 26 & 17 & 25 & 22 & 8 & 2 & - & 27 & 7,5 YR $6 / 2$ \\
\hline \multirow[t]{3}{*}{5} & 28 & 14 & 30 & 26 & 2 & - & - & 22 & 7,5 YR $7 / 2$ \\
\hline & 20 & 18 & 32 & 23 & 5 & 2 & - & 23 & 7,5 YR $6 / 2$ \\
\hline & 20 & 14 & 20 & 22 & 17 & 6 & 2 & 24 & $2,5 \mathrm{Y} 8 / 2$ \\
\hline \multirow[t]{2}{*}{6} & 12 & 9 & 16 & 26 & 26 & 9 & 2 & 18 & $10 \mathrm{YR} 6 / 3$ \\
\hline & 27 & 15 & 22 & 23 & 11 & 2 & 1 & 25 & $10 \mathrm{YR} \mathrm{6/3}$ \\
\hline
\end{tabular}

Grobsandanteil fehlt. Eine ähnliche Zusammensetzung mit etwas höherem Ton-, aber einem stets unter $10 \%$ liegenden Fein- und Mittelsandanteil weisen die Feinsedimente in einem $1,5 \mathrm{~km}$ östlich gelegenen Glacisrest auf (Abb. 1, Aufschluß 5).
Einen weiteren Hinweis auf die äolische Entstehung der beiden letztgenannten Vorkommen geben die gerundeten und mattierten Quarzkörner der Sandfraktion. Die als mittelquartäre Deluviallösse eingeordneten Sedimente haben die charakteristischen Eigen-

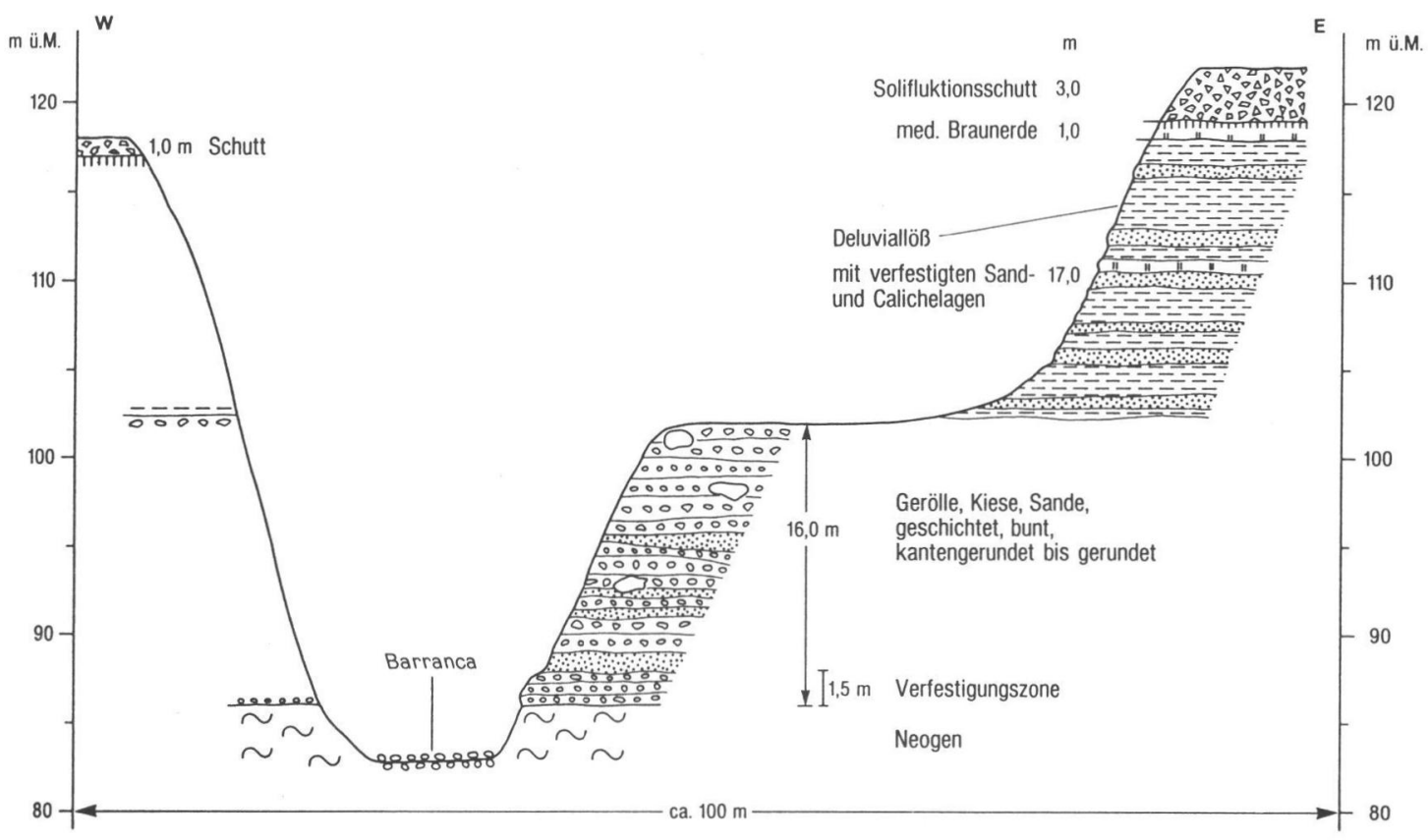

Abb. 4: Mittelquartärer Akkumulationskörper aus fluviatilen, äolischen und periglazialen Ablagerungen — Aufschluß 3. 


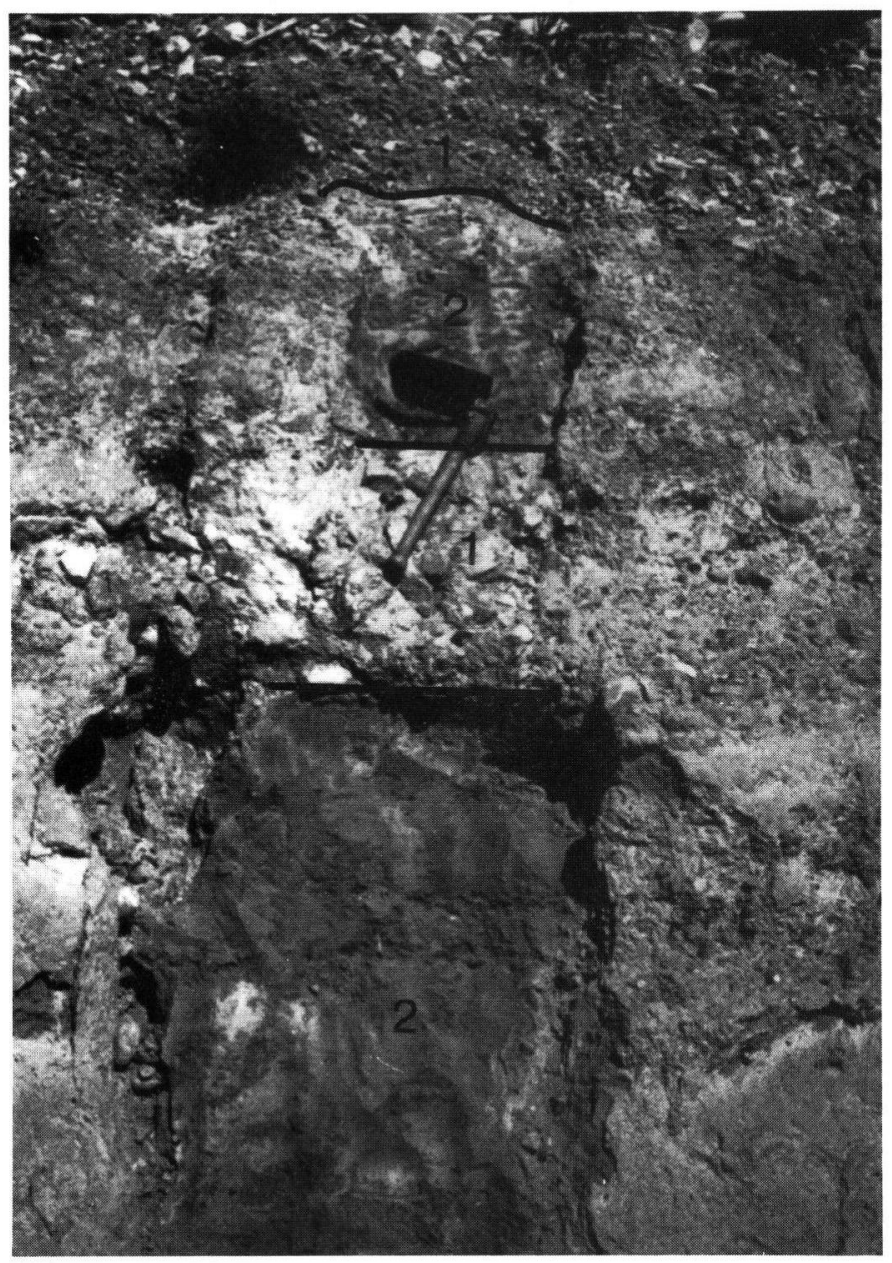

Abb. 5: Wechsellagerung aus Deluviallössen (2) und Solifluktionsschuttdecken (1) - Aufschluß 6 schaften der von BRUNNACKER beschriebenen würmeiszeitlichen Lösse dieses Raumes. So ist die Schwankungsbreite des $\mathrm{CaCO}_{3}$-Gehaltes mit $10-27 \%$ und der Korngrößenzusammensetzung wesentlich größer als in mitteleuropäischen Lössen (BRUNNACKER 1979: 112). Übereinstimmungen liegen auch in der hellgrau-braunen Farbe und der guten Standfestigkeit vor, jedoch weist die Mächtigkeit der mittelquartären Lösse eine größere Schwankungsbreite auf.

Wenn mittelquartäre Deluviallösse bisher aus Südostspanien nicht erwähnt wurden, so mag dies an einem ,nicht austeichend intensiven Suchen nach Lößprofilen" (BROSCHE 1977: 338) liegen oder daran, daß Lösse in kleinsten Inseln vorkommen, „die leicht übersehen werden" (BRUNNACKER 1974: 65).

Für die Auswehung der Feinsedimente, die als Hinweise auf kalt-trockene Verhältnisse zu werten sind, standen neben den breiten Talzügen der Vorfluter auch Teile der Glacisoberflächen selbst zur Verfügung. So treten innerhalb der Glacisakkumulationen mehrere Meter mächtige klastische Sedimente auf (Abb. 6), die aus ungeschichtetem, matrixarmen Schutt bestehen und ebenfalls als Liefergebiet gedient haben können. Hinzu kommt der breite Saum oberhalb des kaltzeitlich abgesenkten Meeresspiegels. Der relativ hohe Fein- und Mittelsandanteil erklärt sich m. E. dadurch, daß aufgrund der Beckenlage die Verwehung nur über wenige Kilometer erfolgte.

Die Deluviallösse bilden stets das Hangende klastischer Ablagerungen und werden ihrerseits immer von Schuttdecken gekappt. Längsachseneinregelungen nach der Methode Poser-Hövermann zeigen für das kantige, ungeschichtete Material einen solifluidalen Transport an. Nur einzelne Lagen sind sortiert und geschichtet. Nach BROSCHE (1978 b: 91) reichen südlich der Sierra Nevada würmeiszeitliche „Schuttdecken mit für Solifluktion sprechendem Einrege- 


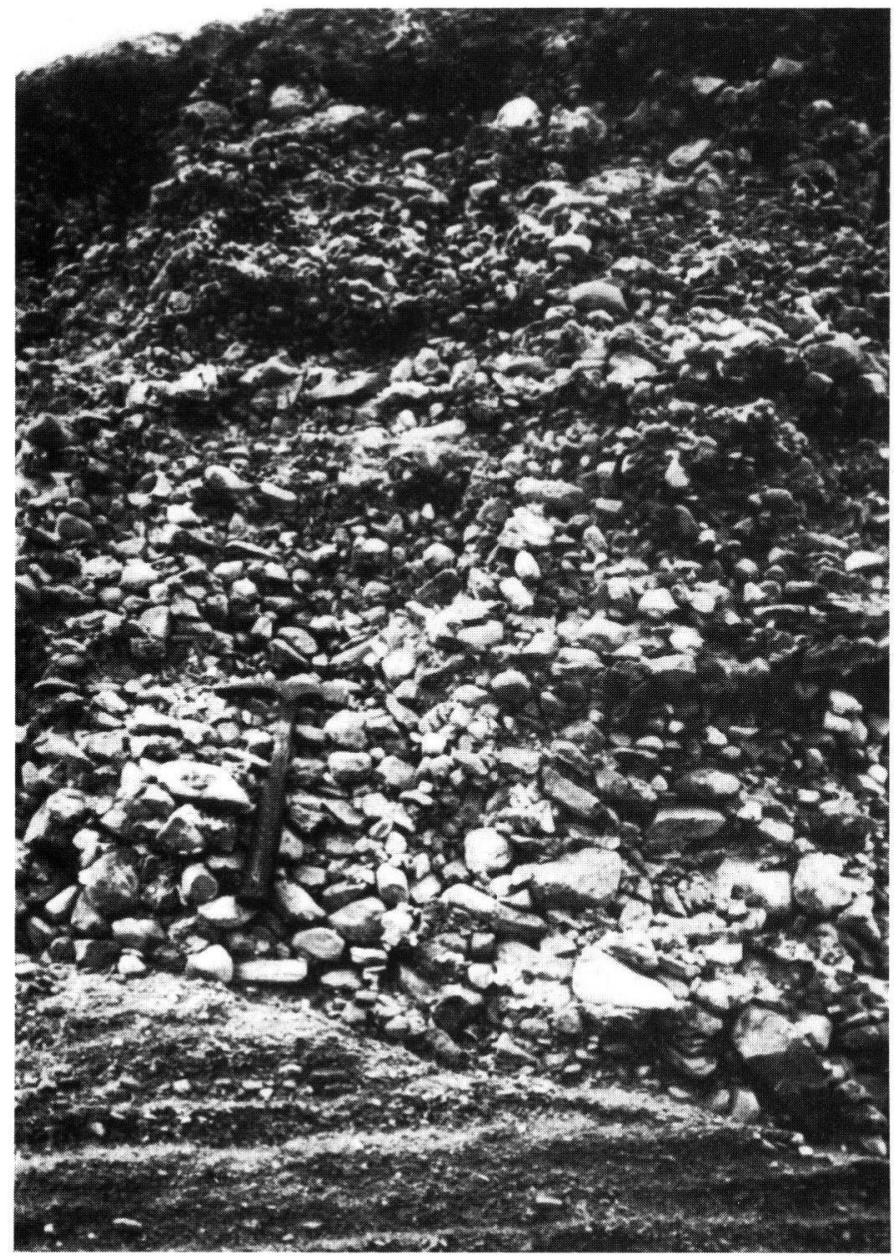

Abb. 6: Matrixarme Glacisschuttdecke.

lungsspektrum ihrer Längsachsen in geschlossenen Vorkommen bis $150-200 \mathrm{~m}$ ü. M. herunter." BRUNNACKER (1974: 65) sieht in ihnen zwar ebenfalls Merkmale intensiver Frostverwitterung, weist jedoch murenartige Bewegungen oder Gekrieche des Gehängespülschuttes nicht gänzlich von der Hand. Aufgrund des geringen Gefälles der Basis der Schuttdecken von weniger als $3^{\circ}$ können im Untersuchungsgebiet Muren ausgeschlossen werden; gegen ein Herabgleiten von wasserübersättigten mergelig-tonigen Sedimenten mit eingelagertem Schutt sprechen sowohl der geringe Feinmaterialanteil als auch die zwischengeschalteten, geschichteten Lagen. Auf einen solifluidalen Transport deuten dagegen auch taschenbodenartige Verwürgungen im Übergangsbereich zu den liegenden, sandigen Schluffen (Abb. 1, Aufschluß 7; Abb. 7) hin. Aus diesen Verwürgungen können keinesfalls Permafrostbedingungen abgeleitet werden. Vielmehr dienen sie als Hinweis auf zumindest zeitweise stark erniedrigte Wintertemperaturen, die einen Wechsel von Gefrier- und Tauprozessen in einem entsprechend tiefen Frostboden anzeigen. Ein Indiz für eine erhebliche Temperaturabsenkung während der Akkumulation der mittelquartären Glacis ist auch ein in einem Grobblocknest enthaltenes, $50 \mathrm{~cm}$ Durchmesser großes Geröll aus schwach verfestigtem Feinsand (Abb. 1, Aufschluß 8; Abb. 8). Da dieses aufgrund seiner Rundung fluviatil transportiert worden ist, muß es im gefrorenen Zustand vorgelegen haben.

Die im Raum Cuevas del Almanzora in mittelquartären Glacisablagerungen enthaltenen Kaltzeitindikatoren, wie mächtige Lößablagerungen mit eingeschalteten Solifluktionsschuttbändern, mehrere Meter mächtiger, solifluidal transportierter Hangschutt, Driftblöcke sowie Sandgerölle weisen auf eine im Vergleich zur Würmkaltzeit größere Temperaturabsenkung hin. Nimmt man wenigstens für einen beschränkten Zeitraum mittlere Wintertemperaturen 


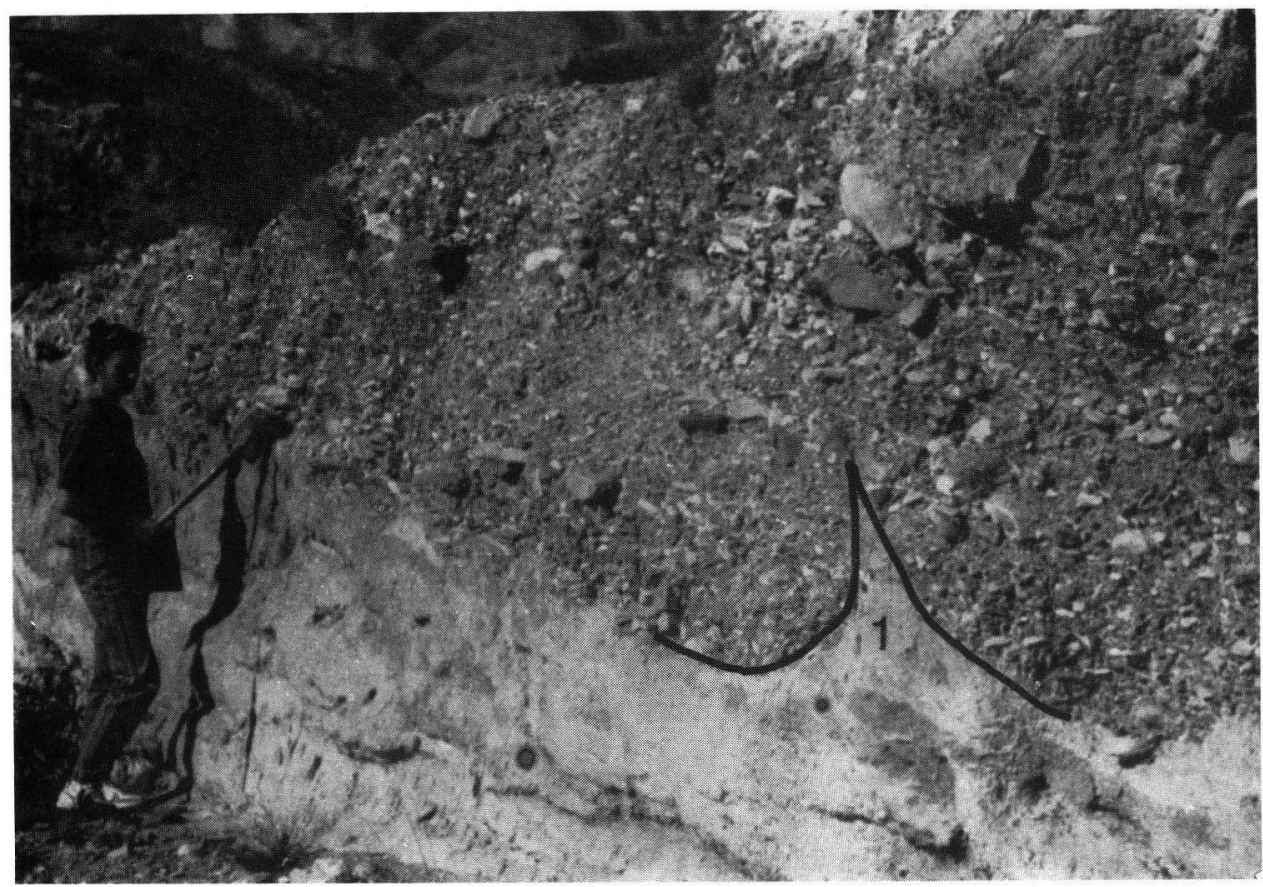

Abb. 7: Solifluktionsschuttdecke mit zwischengeschalteten fluviatilen Schichten und Verwürgungen (1) im Übergangsbereich zu den liegenden sandigen Schluffen - Aufschluß 7.

von $-2^{\circ} \mathrm{C}$ an, so würde sich zumindest für einige Zeitabschnitte der mittelquartären Glacisbildung eine winterliche Temperaturerniedrigung von $12-15^{\circ} \mathrm{C}$ ergeben. Diese Interpretation steht in Einklang mit den Vorstellungen von BUTZER (1964: 21f.), demzufolge die älteren Kaltzeiten im westlichen Mittelmeerraum um ca. $4^{\circ} \mathrm{C}$ kälter waren als die jüngeren. Daß in diesem Raum niedrige Wintertemperaturen auch in der Gegenwart auftreten können, zeigen die absoluten Minima von Murcia. So wurden dort in einem Februar $-10^{\circ} \mathrm{C}$ gemessen, ein Wert, der um mehr als $20^{\circ} \mathrm{C}$ unter der mittleren Februartemperatur liegt (Tab. 1).

BROSCHE (1978a: 192f.) faßt die bisher in der Literatur beschriebenen Kryoturbationserscheinungen in Spanien zusammen. Danach konzentrieren sich die meisten Vorkommen auf mittelquartäre Ablagerungen. Nach LHÉNAFF (1986: 23) gilt das Mittelquartär als eine Periode mit großer morphodynamischer Aktivität, wobei die Glaziale durch Trockenheit und Kälte charakterisiert waren. BOUSQUET (1986: $31 \mathrm{f}$.) dagegen führt die bedeutende Reliefentwicklung dieser Zeit auf eine besonders intensive Tektonik zurück. Hierzu soll im folgenden Stellung genommen und versucht werden, die Bildungszeit des mittelquartären Glacis etwas präziser zu fassen.

\section{Zur Alterseingrenzung der mittelquartären Glacisbildung}

Zwischen der Sedimentation der hangenden Schichten der villafrancazeitlichen Glacisreste, die nordwestlich der "Tres Cabezos" bis maximal $187 \mathrm{~m}$ ü. M. reichen, und der am Steilabfall zum Río Almanzora an der Basis in 80-90 m einsetzenden Geröllakkumulation des mittelquartären Glacis hat im Untersuchungsgebiet eine Phase intensiver Ausräumung stattgefunden, während der Teile der Landoberfläche bis zu $100 \mathrm{~m}$ erniedrigt wurden. Da die Basis der villafrancazeitlichen Glacisreste von den Tres Cabezos zum nördlichen Gebirgstand von $155 \mathrm{~m}$ auf $105 \mathrm{~m}$ (Abb. 1, Aufschluß 9) abnimmt und hier tektonisch gestört ist, ist ein Aufleben der Beckenrandverwerfungen nach Ausbildung des villafrancazeitlichen Glacis als Hauptursache der Ausräumung zu sehen. In dieser Zeit erfolgte der Durchbruch des Río Almanzora durch die Sierra de Almagro (WENZENS 1993, im Druck). Die erst altquartäre Flußablenkung erklärt auch die starke Ausräumung des villafrancazeitlichen Glacis in diesem Abschnitt des Beckens. Angaben über die Zeitdauer und klimatischen Gegebenheiten dieser Erosionsphase, in der nicht nur große Teile des ca. $30 \mathrm{~m}$ mächtigen Akkumulationskörpers des villafrancazeitlichen Niveaus abgetragen und darüber 


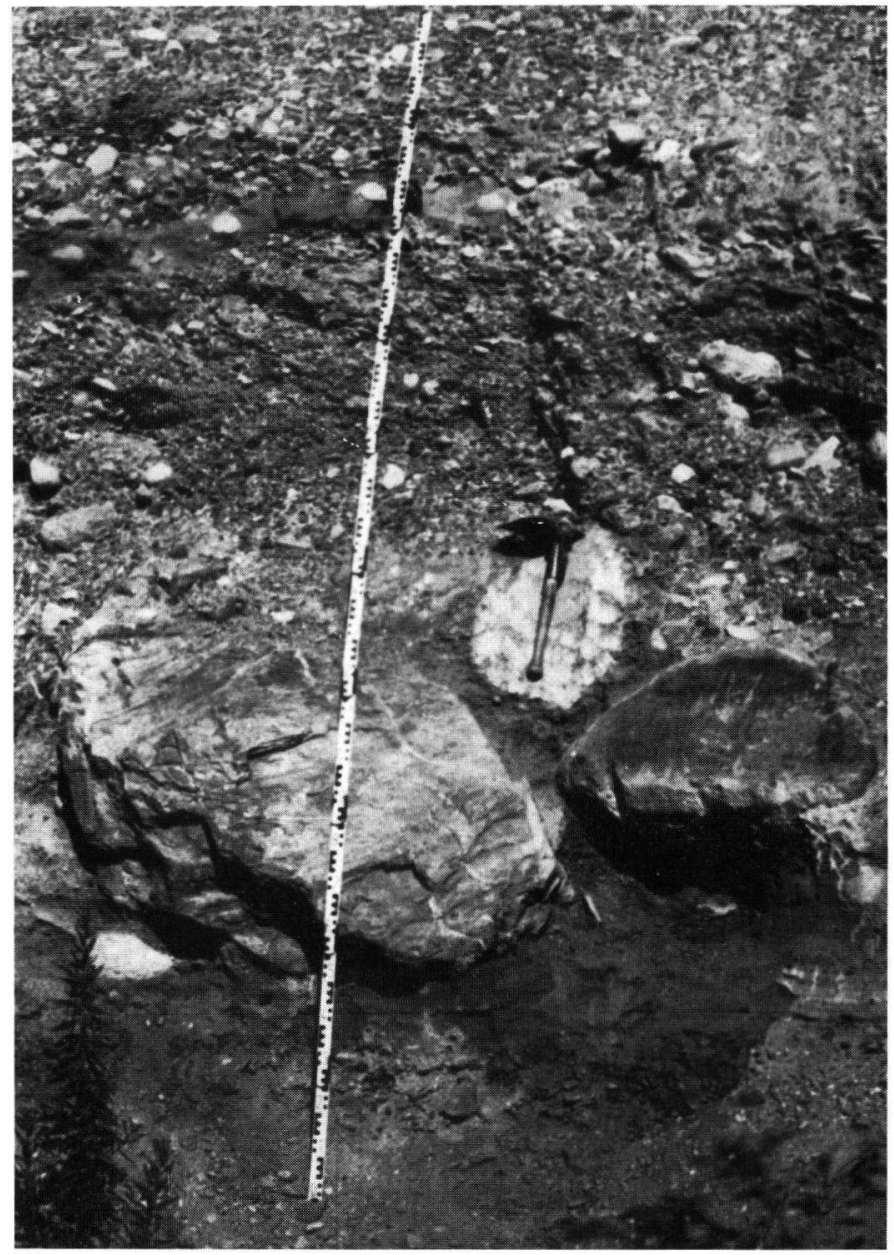

Abb. 8: Fluviatil transportierter Block aus schwach verfestigten Sanden (Spaten) - Aufschluß 8.

hinaus die liegenden Neogenschichten tiefergelegt und größtenteils eingeebnet wurden, können nicht vorgenommen werden. Es empfiehlt sich, diese Ausräumungsphase in das Altquartär zu stellen.

Sieht man von der im gesamten Quartär aktiven Palomares-Verwerfung ab, in deren Nähe das Glacis westlich der Störung aufgewölbt wurde, so sind die mittelquartären Ablagerungen im Untersuchungsgebiet nicht verstellt. Die Akkumulation des mittelquartären Glacis ist daher nicht tektonisch bedingt, wie dies BOUSQUET (1986) annimmt, sondern geht in erster Linie auf eine Zeit mit zumindest teilweise stark abgesenkten Temperaturen und längeren Trockenphasen zurück. Die Akkumulation des mittelquartären Glacis (Abb. 1, Aufschluß 7) schließt mit einer Hangschuttdecke $a b$. Von der ehemaligen Bodenbildung ist nur ein massiver, bis zu $1 \mathrm{~m}$ mächtiger Kalkanreicherungshorizont erhalten. Die ESR-Altersabschätzungen mehrerer Proben liegen alle über $410 \mathrm{ka}$. Im Hangenden befinden sich noch bis zu 3 weitere Cc-Horizonte, die von rubefizierten Sanden, Kiesen und Geröllen getrennt werden.

Der Zeitraum der Glacisbildung läßt sich somit auf das frühe Mittelquartär eingrenzen, wobei nicht auszuschließen ist, daß die Akkumulation der z. T. über $35 \mathrm{~m}$ mächtigen Sedimente (Abb. 4) mehrere Kaltzeiten umfaßte. So können südlich des Río Almanzora drei mittelquartäre Glacis-Niveaus ausgegliedert werden (WENZENS 1991, 190f). Dies gilt auch für das Becken von Huércal-Overa, wo ebenfalls bis zu drei mittelquartäre Glacis auftreten (WENZENS 1993).

Östlich von Cuevas del Almanzora ist mit der Entstehung dieses Glacis die Bildung weitflächiger Verebnungen Vera abgeschlossen. Im Zuge der Eintiefung des Río Almanzora und seiner Nebenflüsse in dieses Niveau wird das Talnetz festgelegt. Im weiteren Verlauf führt der Wechsel zwischen Akkumulation und Erosion zur Ausbildung von Flußterrassen. So ist dem mittelquartären Glacis bei La Portilla (Abb. 1, 
Aufschluß 10) eine Flußterrasse in $90 \mathrm{~m}$ ü. M. vorgelagert. Die insgesamt $16 \mathrm{~m}$ mächtige Aufschüttung besteht im wesentlichen aus gut geschichteten und sortierten Kiesen und Geröllen. Im Unterschied zur Zusammensetzung der Glacissedimente erreichen die Grobblöcke maximal 1,5 m Durchmesser und sind gerundet.

Reste dieses Niveaus treten vor allem auf der rechten Talseite auf, wo noch zwei weitere Terrassenniveaus ausgegliedert werden können. Sie weisen eine wesentlich geringere Mächtigkeit auf und lassen sich bis zur Palomares-Verwerfung als kleinräumige Relikte verfolgen. Die letzteiszeitliche Flußterrasse befindet sich am Eintritt des Río Almanzora nur wenige Meter über dem breiten rezenten Talboden und taucht im Mündungsbereich unter die holozänen Ablagerungen ab. Diese Sedimente zeichnen sich durch einen hohen Kies- und Sandanteil aus, Grobgerölle treten stark zurück, und die für kaltzeitliche Ablagerungen charakteristische Inkrustierung fehlt.

\section{Schriftenverzeichnis}

BLÜMEL, W. (1981): Pedologische und geomorphologische Aspekte der Kalkkrustenbildung in Südwestafrika und Südostspanien. - Karlsruher Geogr. Hefte, 10: 228 S., 51 Abb., 8 Karten, 6 Tab.; Karlsruhe.

BousqueT, B. (1986): Tectonique et quaternaire moyen en Méditerranée. - Bull. Assoc. Géogr. Franc.: 31-44, 1 Abb., 1 Tab.; Paris.

BRosCHE, K. (1977): Geomorphologische und bodengeographische Analyse holozäner, jung- und mittelpleistozäner Sedimente und Böden in Spanien und Südfrankreich. - Catena, 3: 311-342, 9 Abb.; Gießen.

- (1978a): Der vorzeitliche periglaziale Formenschatz auf der Iberischen Halbinsel, Möglichkeiten zu einer klimatischen Auswertung. - Colloq. sur le Périglaciaire d'Altitude du dom. Méditer. et abords.: 187-202, $1 \mathrm{Abb}$; Strasbourg.

- (1978b): Ergebnisse einer vergleichenden Studie zum rezenten und vorzeitlichen periglazialen Formenschatz auf der Iberischen Halbinsel. - Biuletyn Peryglacyalny, 27: 53-103, 16 Abb., 4 Fig.; 2 Tab.; Lódz.

- (1978c): Beiträge zum rezenten und vorzeitlichen periglazialen Formenschatz auf der Iberischen Halbinsel. Abhandl. Geogr. Inst., Sonderh., 1: 287 S., 13 Abb., 19 Tab., 61 Phot., 2 Karten; Berlin.

BRUNNACKeR, K. (1974): Lösse und Paläoböden der letzten Kaltzeit im mediterranen Raum. - Eisz. u. Gegenw., 25: 62-95, 10 Abb., 3 Tab.; Öhringen.

- (1979): Zur Bodengeschichte des Jungquartärs im mediterranen Raum. - Z. Geom. N.F., Suppl.-Bd., 33: 109-117, 3 Abb.; Berlin.

— \& LOŽEK, V. (1969): Lößvorkommen in Südostspanien. - Zeitschr. f. Geom. N. F., 13: 297-316, 5 Abb., 2 Tab.; Berlin.
BUTZER, K. (1964): Pleistocene cold-climate phenomena of the island of Mallorca. - Zeitschr. f. Geom. N. F., 8: 7-31; Berlin.

Couvreur, G. \& RAYNAL, R. (1981): Quelques thèmes de recherche géomorphologique dans les pays méditerranéens. - Mediterranée, 4: 43-51; Aix-en-Prov.

Dumas, B. (1979): Variations climatiques quaternaires et morphogenèse dans le Sud du Levant espagnol. Médit., 3: 27-36, 6 Abb.; Aix-en-Prov.

Ferre Bueno, E. (1979): El valle del Almanzora. - 494 S., 80 Abb., 27 Tab., 44 Phot.; Almería.

HaRveY, A. (1978): Dissected alluvial fans in southeast Spain. - Catena, 5: 177-211, 14 Abb., 7 Phot.; Braunschweig.

- (1987): Patterns of Quaternary aggradational and dissectional landform development in the Almería region, southeast Spain: a dry-region, tectonically active landscape. - Die Erde, 118: 193-215, 9 Abb., 1 Tab.; Berlin.

HoffmanN, G. (1988): Holozänstratigraphie und Küstenlinienverlagerung an der andalusischen Mittelmeerküste. - Berichte Fachber. Geowissensch. Univ. Bremen, 2: 173 S., 47 Abb., 21 Tab.; Bremen.

LHÉNAFF, R. (1986): Héritages morphoclimatiques et environnements du quaternaire moyen méditerranéen. - Bull. Assoc. Géogr. Franc.: 21-27; Paris.

MỨler, M. (1979): Handbuch ausgewählter Klimastationen der Erde. - Forschungsstelle Bodenerosion der Univ. Trier, 5: 346 S., 10 Karten; Trier.

Thornes, J. (1976): Semi-arid erosional systems. Geogr. Papers, 7; London.

VÖLK, H. (1967): Zur Geologie und Stratigraphie des Neogenbeckens von Vera, Südost-Spanien. - Diss. Univ. Amsterdam, 160 S., 3 Abb., 2 Tab., 8 Phot.; Wageningen.

- (1973): Klima und Vegetation im Mündungsgebiet des Río Almanzora und Río de Aguas. - Heidelb. Geogr. Arb., 38: 267-282, 2 Abb., 4 Tab.; Heidelberg.

- VÖLK, H. (1979): Quartäre Reliefentwicklung in Südost-Spanien. - Heidelb. Geogr. Arb., 58: 143 S., 11 Abb., 28 Phot., 1 Karte; Heidelberg.

WENZENS, G. (1991): Die mittelquartäre Reliefentwicklung am Unterlauf des Río Almanzora (Südostspanien). Freiburger Geogr. Hefte, 33: 185-197, 11 Abb.; Freiburg.

- (1992): The influence of tectonics and climate on the Villafranchian morphogenesis in semiarid Southeastern Spain. - Zeitschr. f. Geom. N.F. Suppl.-Bd. 84: 173-184, 9 Abb., 2 Phot., 1 Tab.; Berlin.

- (1993): The influence of Quaternary tectonics on river capture and drainage patterns in the Huércal-Overa basin, SE-Spain. Balkema (im Druck).

- \& RommerSKIRCHEN, E. (1979): Zur genetischen Differenzierung von Fußflächen im Bereich der Südmeseta. - Landschaftsgenese u. Landschaftsökologie, 5: 101-104; Braunschweig.

Manuskript eingegangen am 13. 12. 1990. 